\title{
Acknowledgement to Reviewers of Dentistry Journal in 2019
}

Dentistry Journal Editorial Office

MDPI, St. Alban-Anlage 66, 4052 Basel, Switzerland

Published: 19 January 2020

The editorial team greatly appreciates the reviewers who have dedicated their considerable time and expertise to the journal's rigorous editorial process over the past 12 months, regardless of whether the papers are finally published or not. In 2019, a total of 116 papers were published in the journal, with a median time to first decision of 23 days and a median time from submission to publication of 69 days. The editors would like to express their sincere gratitude to the following reviewers for their generous contribution in 2019:

\begin{tabular}{ll} 
Abdelaziz, Marwa & Baghdadi, Ziad D. \\
Abduo, Jaafar & Bahari-Chopiuk, Nasrin \\
Abrams, Stephen & Baltriukiene, Daiva \\
Abubakr, Neamat Hassan & Banas, Jeffrey \\
Adekoya, Timothy O. & Barbeck, Mike \\
Afrashtehfar, Kelvin & Barberis, Fabrizio \\
Afrashtehfar, Kelvin Ian & Barnett, Tony \\
Agrawal, Priyanka & Batchelor, Paul \\
Aimetti, Mario & Bekes, Katrin \\
Akinkugbe, Aderonke A. & Bel'skaya, Lyudmila \\
Aksel, Hacer & Bernardello, Fabio \\
Al-Horani, Rami A. & Bernardi, Sara \\
Almståhl, Annica & Bettini, Giordana \\
Al-Rudainy, Dhelal & Bian, Li \\
Anastasiou, Antonios & Borzabadi-Farahani, Ali \\
Andrukhov, Oleh & Bourgeois, Denis \\
Angelova Volponi, Ana & Brantley, William \\
Arany, Praveen & Bright, Richard \\
Ardelean, Lavinia Cosmina & Britt, Eileen \\
Arendarczyk, Natalia & Browar, Andrew W. \\
Arora, Himanshu & Buckley, M. \\
Asa'ad, Farah & Bullon, Pedro \\
Aspriello, Simone Domenico & Calvo-Guirado, José Luis \\
Assari, Shervin & Campus, Guglielmo \\
Avent, Minyon & Carey, Clifton \\
Ayoub, Ashraf & Carrilho, Eunice \\
Ayuso-Montero, Raúl & Carrouel, Florence \\
Casimiro, Maria Helena & El-Bialy, Tarek \\
\hline &
\end{tabular}


Cerra, Frank

Cervino, Gabriele

Chacko, Jenu Varghese

Chang, Meichi

Chen, Junning

Chen, Min-Huey

Cheung, Gary Shun Pan

Chi, Gerald

Chladek, Grzegorz

Choi, Sung-Hwan

Chrysanthakopoulos, Nikolaos A.

$\mathrm{Chu}$, Chun Hung

Cicciù, Marco

Coleman, Nichola

Coletta, Ricardo

Contaldo, Maria

Corbella, Stefano

Cui, Zhibin

Cvikl, Barbara

Cvikl, Doz. Barbara

Czarnecka, Beata

Dalessandri, Domenico

Dammaschke, Till

Danesh-Sani, Seyed Amir

Darvell, Brian

De Biase, Alberto

Delimont, Nicole

Dellavia, Claudia

Derman, Sonja H. M.

Di Blasio, Alberto

Di Carlo, Gabriele

Di Stasio, Dario

Diogo, Patrícia

Dioguardi, Mario

Diomede, Francesca

Dionysopoulos, Dimitrios

Doerrler, William

Donchin, Milka

Dziedzic, Arkadiusz

Ehrlich, Ygal

Eick, Sigrun

Ekuni, Daisuke

Hanisch, Marcel
El-Housseiny, Azza

Eliades, George

Eliades, Theodore

Emmanouil, Dimitris

Enrico, Gherlone Felice

Fakhouri, Walid D.

Farronato, Davide

Felix Gomez, Grace Gomez

Fernandes, Maria Goreti

Fernández-Revelles, Andrés B.

Ferrarotti, Francesco

Ferreira, Manuel Marques

Figueiral, Maria Helena

Finer, Yoav

Fiorillo, Luca

Flaherty, Kevin

Fornaini, Carlo

Forner, Leopoldo

Foster Page, Lyndie A.

Fox, Simon

França, Rodrigo

Fu-Mei, Huang

Fusco, Vittorio

Galal, Suzanne

Galli, Silvia

Galluccio, Gabriella

Gao, Xin

Garner, Angie

Ghezzi, Elisa M.

Gibson, Frank

Glavina, Domagoj

Glazar, Irena

Glockner, Karl

Gorseta, Kristina

Goto, Takaharu

Gregory, Richard L

Grimm, Wolf-Dieter

Grzech-Leśniak, Kinga

Gudey, Shyam Kumar

Gutierrez-Perez, Jose-Luis

Hack, Gary

Han, Pingping

Kontonasaki, Eleana 
Haraszthy, Violet

Haynes, David

Hemphill, Jean Croce

Herten, Monika

Heyman, Richard E

Hirata, Ronaldo

Hoover, Jay

Horowitz, Robert A.

Howe, Brian

Hristov, Ilian

Hughes, Christopher V.

Hülsmann, Michael

Ingendoh-Tsakmakidis, Alexandra

Innes, Nicola

Irwin, Jennifer

Isola, Gaetano

Janakiraman, Harinarayanan

Jeng, Jiiang-Huei

Jinno, Yohei

Johnson, Maree

Jordana, Fabienne

Joseph, Susan

Ju, Xiangqun

Kaisarly, Dalia

Kameyama, Atsushi

Kaminski, Rafal

Kanamoto, Taisei

Kanno, Takahiro

Kanzaki, Hiroyuki

Kanzow, Philipp

Karpiński, Tomasz M

Katic, Visnja

Kebschull, Moritz

Khurshid, Zohaib

Kim, Hae-Young

Kim, J.-W.

Kim, Jin-Bom

Kim, Seong-Gon

Kim, Young-jae

Klontzas, Michael

Kniha, Kristian

Marto, Carlos Miguel

Mascitti, Marco
Korsunsky, Alexander M.

Kosuke, Nozaki

Kranz, Stefan

Krystofiak, Tomasz

Kujan, Omar

Kumar, PRAKASH

Kusnoto, Budi

Kwon, Tae-Yub

Lambert, Martijn

Łapińska, Barbara

Laranjo, Mafalda

Larsson, Christel

Lauritano, Dorina

Lee, Cheng-I

Lee, Sang Jin

Lee, Sung-Hoon

Lerner, Henriette

Lezot, Frederic

Li, Jiao Jiao

Lin, Hai-Shu

Lin, Yuh-Yih

Litschauer, Brigitte

Liu, Yu

Lo Giudice, Giuseppe

Lo Muzio, Lorenzo

Loewy, Zvi

Lombardi, Tommaso

Lombardo, Giorgio

Lopez-Jornet, Pia

Lukomska-Szymanska, Monika

Lunov, Oleg

Ma, Sunyoung

Maddi, Abhiram

Magán-Fernández, Antonio

Magiorkinis, Emmanouil

Maiorana, Carlo

Makvandi, Pooyan

Manton, David

Marcal, Helder

Marcé-Nogué, Jordi

Marshman, Zoe

Martins, Francisco

Popat, Hashmat 


\begin{tabular}{|c|c|}
\hline Matys, Jacek & Porcheri, Cristina \\
\hline Maurin, Jean-Christophe & Porojan, Liliana \\
\hline Mayberry, Melanie & Porritt, Jenny \\
\hline Mays, Jacqueline W. & Pramudita, Jonas A. \\
\hline Meister, Jörg & Qasim, Saad \\
\hline Mendes, Joaquim & Quinn, Barry \\
\hline Merigo, Elisabetta & Ramaswamy, Ravishankar \\
\hline Mertas, Anna & Randall, Cameron \\
\hline Meurman, Jukka & Read-Fuller, Andrew \\
\hline Mezzomo Collares, Fabrício & Reddy, Sakamuri \\
\hline Micutz, Marin & Rener-Sitar, Ksenija \\
\hline Mihelogiannakis, Dimitrios & Reukov, Vladimir \\
\hline Mitsea, Anastasia & Reychler, Hervé \\
\hline Mobilio, Nicola & Ribeiro, Apoena \\
\hline Moore, Rod & Ríos-Santos, Jose Vicente \\
\hline Morra, Marco & Ritz, Ulrike \\
\hline Moussa, Dina & Robertson, Agneta \\
\hline Nalliah, Romesh & Rodríguez-Lozano, Francisco Javier \\
\hline Nicholi Vorsa, Nicholi Vorsa & Roehling, Stefan K. \\
\hline Nicolescu, Mihnea-Ioan & Rogers, John \\
\hline Nieminen, Pentti & Roggendorf, Matthias J \\
\hline Ogi, Kazuhiro & Romano, Federica \\
\hline Ogórek, Rafał & Rongo, Roberto \\
\hline Owens, Janine & Rudolf, Rebeka \\
\hline Padial-Molina, Miguel & Rudolph, Heike \\
\hline Pagano, Stefano & Rusu, Laura Cristina \\
\hline Palma, Paulo J & Ruys, Andrew \\
\hline Palmer, Robert J. & Ryabov, Igor \\
\hline Palomo, Leena & Saadi, Irfan \\
\hline Pang, Eun-Kyoung & Sabbagh, Heba Jafar \\
\hline Papadopoulos, Moschos & Safavi, Kamran \\
\hline Papadopoulou, Alexandra & Sahrmann, Philipp \\
\hline Par, Matej & Sato, Takuichi \\
\hline Park, Chan Ho & Schiavino, Domenico \\
\hline Park, Ji-Man & Scoffield, Jessica \\
\hline Park, Jun-Beom & Scribante, Andrea \\
\hline Patel, Upen & Sefat, Farshid \\
\hline Pathak, Ashish & Segura, Juan-Jose \\
\hline Patini, Romeo & Sekiguchi, Yu \\
\hline Peroš, Kristina & Seok, Hyun \\
\hline Pilo, Raphael & Serpico, Rosario \\
\hline Shahmoradi, Mahdi & Shah, Parag K \\
\hline Shanmugam, Muruganandan & Vassiliou, Leandros-Vassilios \\
\hline
\end{tabular}


Short, Leonie

Singha, Subhankar

Singhrao, Sim K.

Sips, Patrick

Sivasubramanian, Kathyayini

Skopouli, Fotini

Sones, Amerian D.

Spagnuolo, Gianrico

Srinivasan, Sowmya

Staderini, Edoardo

SUKUMARAN, ANIL

Sung, Wen-Wei

Surdacka, Anna

Surdacki, Andrzej

Suzuki, Jon B.

Swain, Michael

Swann, Brian Jeffrey

Szczepanski, Caroline

Tallarico, Marco

Testori, Tiziano

Thikkurissy, Sarat

Thorsen, Andreas

Timothe, Peggy

Tomaszewska, Ewa

Torres-Lagares, Daniel

Troedhan, Angelo

Tseng, Yu-Chuan

Tsuchida, Sachio

$\mathrm{Tu}$, Ming-Gene

Tuna, Taskin

Upadhyaya, Jasbir

Vandenberghe, Bart
Vaz, Maria De Fátima Reis

Vaz, Paula

Veerkamp, Jaap

Verket, Anders

Vettore, Mario

Vissink, Arjan

Vozza, Iole

Walsh, Laurence J

Walter, Christian

Wang, Haoyu

Wang, Jeff CW.

Wang, Kai

Washio, Ayako

White, Raymond P.

Wieckiewicz, Mieszko

Wierichs, Richard

Wilson, Michael

Wunsch, Patrice B.

Xiao, Li

Xie, Qian

Yamagata, Kenji

Yamaguchi, Satoshi

Yang, Chengwu

Yeh, Chih-Ko

Yeung, Andy Wai Kan

Yuan, Kuo

Yuan, Siyang

Yumoto, Hiromichi

Zafar, Muhammad Sohail

Zhan, Ling

Ziebart, Thomas 